\title{
The Effect of Holder Pasteurization on Nutrients and Biologically-Active Components in Donor Human Milk: A Review
}

\author{
Chiara Peila ${ }^{1, *}$, Guido E. Moro ${ }^{2}$, Enrico Bertino ${ }^{1}$, Laura Cavallarin ${ }^{3}$, Marzia Giribaldi ${ }^{3,4}$, \\ Francesca Giuliani ${ }^{1}$, Francesco Cresi $^{1}$ and Alessandra Coscia ${ }^{1}$ \\ 1 Complex Structure Neonatology Unit, Department of Public Health and Pediatric, University of Turin, \\ Via Ventimiglia 3, Torino 10126, Italy; enrico.bertino@unito.it (E.B.); quo.g@libero.it (F.G.); \\ fcresi@gmail.com (F.C.); alessandra.coscia@unito.it (A.C.) \\ 2 Italian Association of Human Milk Banks, Via Libero Temolo 4, Milan 20126, Italy; guidoemoro@tiscali.it \\ 3 Institute of Sciences of Food Production, National Research Concil, largo Braccini 2, Grugliasco 10095, Italy; \\ laura.cavallarin@ispa.cnr.it (L.C.); marzia.giribaldi@crea.gov.it (M.G.) \\ 4 Food \& Nutrition Research Center, Council for Agricultural Research and Economics, via Ardeatina 546, \\ Roma 00178, Italy \\ * Correspondence: peila.chiara@gmail.com; Tel.: +39-11-313-4438; Fax: +39-11-313-4612
}

Received: 9 May 2016; Accepted: 26 July 2016; Published: 2 August 2016

\begin{abstract}
When a mother's milk is unavailable, the best alternative is donor milk (DM). Milk delivered to Human Milk Banks should be pasteurized in order to inactivate the microbial agents that may be present. Currently, pasteurization, performed at $62.5^{\circ} \mathrm{C}$ for $30 \mathrm{~min}$ (Holder Pasteurization, HoP), is recommended for this purpose in international guidelines. Several studies have been performed to investigate the effects of $\mathrm{HoP}$ on the properties of DM. The present paper has the aim of reviewing the published papers on this topic, and to provide a comparison of the reported variations of biologically-active DM components before and after HoP. This review was performed by searching the MEDLINE, EMBASE, CINHAL and Cochrane Library databases. Studies that clearly identified the HoP parameters and compared the same DM samples, before and after pasteurization, were focused on. A total of 44 articles satisfied the above criteria, and were therefore selected. The findings from the literature report variable results. A possible explanation for this may be the heterogeneity of the test protocols that were applied. Moreover, the present review spans more than five decades, and modern pasteurizers may be able to modify the degradation kinetics for heat-sensitive substances, compared to older ones. Overall, the data indicate that HoP affects several milk components, although it is difficult to quantify the degradation degree. However, clinical practices demonstrate that many beneficial properties of DM still persist after HoP.
\end{abstract}

Keywords: human milk; donor milk; holder pasteurization; Human Milk Banks

\section{Introduction}

Human milk (HM) is the gold standard for the feeding and nutrition of preterm and term newborns [1-3]. A mother's own milk is the first choice for improving the short- and long-term outcomes for all neonates [1]. However, the benefits of $\mathrm{HM}$ are mediated by several specific bioactive and immunomodulatory factors, and HM can be considered a species-specific biological "dynamic" system [4].

When a mother's own milk is unavailable or in short supply (a common occurrence in Neonatal Intensive Care Units), the World Health Organization and the American Academy of Pediatrics recommend the use of donor milk (DM) as the best alternative [1,3]. Milk delivered to Human Milk Banks (HMBs) should be pasteurized so as to inactivate viral and bacterial agents [5]. The ideal 
pasteurization process should consist of a phase of rapid heating, followed by a phase in which the temperature is maintained constant, and a final phase of rapid cooling. Currently, a pasteurization process at $62.5{ }^{\circ} \mathrm{C}$ for $30 \mathrm{~min}$ (the Holder pasteurization method, HoP) is recommended in all international guidelines for the constitution of HMBs [5,6]. Pasteurized milk is known to retain many beneficial and protective effects of HM. This method is thought to lead to a good compromise between the microbiological safety and nutritional/biological quality of DM [7-11].

Nonetheless, it is also well known that $\mathrm{HoP}$ affects some of the nutritional and biological properties of HM. Several studies have been performed to investigate the effects of Holder pasteurization on the nutritional and immunological properties of DM, but a comprehensive review and comparison of the related results is, to the best of the authors' knowledge, not available in literature. Currently, the available data consist of reviews regarding the advantages of donor milk, but very few details are given on the effects of HoP on the nutrients of mother's milk [12-16].

Thus, the present paper is aimed at reviewing the published papers, and at comparing the results related to the effects of HoP on the biological and nutritive components of DM.

\section{Search Methodology}

The literature review was performed by conducting electronic searches of MEDLINE, EMBASE, CINHAL and the Cochrane Library. The electronic search used the following keywords and MeSH terms: (i) donor milk; (ii) banked milk; (iii) milk bank; (iv) milk banking; (v) (human milk OR donor milk) AND Holder pasteurization; (vi) (human milk OR donor milk) AND pasteurization; (vii) (human milk OR donor milk) AND storage; (viii) (human milk OR donor milk) AND heat treatment; (ix) (donor milk OR Holder pasteurization) AND protein; (x) (donor milk OR Holder pasteurization) AND enzyme; (xi) (donor milk OR Holder pasteurization) AND lipid; (xii) (donor milk OR Holder pasteurization) AND saccharide; (xiii) (donor milk OR Holder pasteurization) AND vitamin; (xiv) (donor milk OR Holder pasteurization) AND minerals; (xv) (donor milk OR Holder pasteurization) AND oxidative stress; (xvi) (donor milk OR Holder pasteurization) AND cytokine; (xvii) (donor milk OR Holder pasteurization) AND hormone; (xviii) (donor milk OR Holder pasteurization) AND growth factor; and (xix) (donor milk OR Holder pasteurization) AND immunoglobulin. No limits concerning the publication date were set.

Considering differences between the research protocols published to date, we focused the review on studies with an experimental design that:

- defined exactly the pasteurization method precisely $\left(62.5-63^{\circ} \mathrm{C}\right.$ for $\left.30 \mathrm{~min}\right)$; and

- compared the same samples of HM before and after the heat treatment.

In order to limit bias in the inclusion/exclusion process, the selection was made with the consensus of two authors (CP and MG).

\section{Results}

A total of 58 articles were found from a combination of the searches, but only 44 fulfilled all the inclusion criteria. Table 1 summarizes the technical details on the methodological aspects of the selected articles. Tables 2-4 provide an overview of the literature on the effects of HoP on different components of DM. 
Table 1. Materials and Methods of the different studies included in the survey.

\begin{tabular}{|c|c|c|c|c|c|c|c|c|}
\hline $\operatorname{Ref} *$ & Preterm/Term & $\begin{array}{l}\text { Phase of } \\
\text { Lactation }\end{array}$ & $\begin{array}{l}\text { Expression } \\
\text { Method }\end{array}$ & Status & $\begin{array}{l}\text { Pre-Pasteurization } \\
\text { Storage }\end{array}$ & $\begin{array}{l}\text { Pasteurization } \\
\text { Equipment }\end{array}$ & Sample Size & Analytical Method ${ }^{\circ}$ \\
\hline \multirow{6}{*}[17]{} & \multirow{6}{*}{$\mathrm{N} / \mathrm{A}$} & \multirow{6}{*}{ Mature } & \multirow{6}{*}{$\mathrm{N} / \mathrm{A}$} & \multirow{6}{*}{ Frozen } & \multirow{6}{*}{$\begin{array}{l}-20^{\circ} \mathrm{C} \text { up to } \\
6 \text { months; thawing } \\
\text { in a water bath at } \\
37.5^{\circ} \mathrm{C}\end{array}$} & \multirow{6}{*}{$\begin{array}{l}\text { Sterifeed: } \\
\text { pre-heated water } \\
\text { bath }\left(63.2^{\circ} \mathrm{C}\right) ; \\
62.5^{\circ} \mathrm{C} \text { for } 30^{\prime} ; \\
\text { cooling in cold } \\
\text { water bath }\end{array}$} & \multirow{6}{*}{$\begin{array}{l}17 \text { pools }-4 \text { donors } \\
\text { each }\end{array}$} & Adiponectine: RIA \\
\hline & & & & & & & & $\begin{array}{l}\text { Insulin: electrochemiluminescence } \\
\text { immunoassay }\end{array}$ \\
\hline & & & & & & & & Total fat: creamatocrit \\
\hline & & & & & & & & Total protein: BCA \\
\hline & & & & & & & & Total energy: bomb calorimetry \\
\hline & & & & & & & & Glucose: enzymatic method \\
\hline [18] & $\begin{array}{l}\text { Preterm and } \\
\text { Term }\end{array}$ & Mature & $\begin{array}{l}\text { Hand or } \\
\text { electric/manual } \\
\text { pump }\end{array}$ & Frozen & $\begin{array}{l}-20^{\circ} \mathrm{C} \text { until } \\
\text { processing; thawing } \\
\text { and heating to } 40^{\circ} \mathrm{C} \\
\text { using a thermostatic } \\
\text { bath }\end{array}$ & $\begin{array}{l}62.5^{\circ} \mathrm{C} \text { for } 30^{\prime} ; \\
\text { cooling to }<4{ }^{\circ} \mathrm{C} \\
\text { in stirred } \\
\text { thermostatic baths }\end{array}$ & $\begin{array}{l}34 \text { samples }-28 \\
\text { donors }\end{array}$ & Infrared Analyzer (MIRIS) \\
\hline [19] & $\mathrm{N} / \mathrm{A}$ & $\mathrm{N} / \mathrm{A}$ & $\begin{array}{l}\text { Hand or } \\
\text { electric/manual } \\
\text { pump }\end{array}$ & Fresh & No & $62.5^{\circ} \mathrm{C}$ for $30^{\prime}$ & 57 samples & $\begin{array}{l}\text { Infrared Analyzer (Milko-scan } \\
\text { Minor) }\end{array}$ \\
\hline \multirow{3}{*}{ [20] } & \multirow{3}{*}{$\begin{array}{l}\text { Preterm and } \\
\text { Term }\end{array}$} & \multirow{3}{*}{ Colostrum } & \multirow{3}{*}{ Hand } & \multirow{3}{*}{ Fresh } & \multirow{3}{*}{ No } & \multirow{3}{*}{$62.5^{\circ} \mathrm{C}$ for $30^{\prime}$} & \multirow{3}{*}{$\begin{array}{l}36 \text { samples: }<32 \\
\text { weeks; } 32 \text { samples: } \\
\text { 32-36 weeks; } 33 \\
\text { samples: >36 weeks }\end{array}$} & Total protein: refraction index \\
\hline & & & & & & & & Lysozyme: lysoplate method \\
\hline & & & & & & & & Immunoglobulins: RIA \\
\hline \multirow{3}{*}[21]{} & \multirow{3}{*}{ Term } & \multirow{3}{*}{ N/A } & \multirow{3}{*}{$\mathrm{N} / \mathrm{A}$} & \multirow{3}{*}{ Fresh } & \multirow{3}{*}{ No } & \multirow{3}{*}{$\begin{array}{l}\text { LABU-Muttermilch } \\
\text { pasteurizer: } \\
62.5^{\circ} \mathrm{C} \text { for } 30^{\prime}\end{array}$} & \multirow{3}{*}{$\begin{array}{l}4 \text { Samples-2 } \\
\text { CMV-positive and } 2 \\
\text { CMV-negative donors }\end{array}$} & $\begin{array}{l}\text { Total protein, alkaline phosphatase } \\
\text { and lipase activity: Hitachi } 917 \\
\text { Automatic Analyzer }\end{array}$ \\
\hline & & & & & & & & $\begin{array}{l}\text { Folic acid, Vitamin B12: } \\
\text { chemiluminescence immunoassays }\end{array}$ \\
\hline & & & & & & & & sIgA and lysozyme: RIA \\
\hline \multirow{2}{*}{ [22] } & \multirow{2}{*}{ N/A } & \multirow{2}{*}{ Mature } & \multirow{2}{*}{ Electric pump } & \multirow{2}{*}{ Fresh } & \multirow{2}{*}{ No } & \multirow{2}{*}{$\begin{array}{l}\text { VLM } \\
\text { exchangeable } \\
\text { HBV-Q-16-16: } \\
63^{\circ} \mathrm{C} \text { for } 30^{\prime}\end{array}$} & \multirow{2}{*}{$\begin{array}{l}30 \text { samples-30 } \\
\text { donors }\end{array}$} & Lysine content: fluorimetry \\
\hline & & & & & & & & Total protein: Lowry method \\
\hline
\end{tabular}


Table 1. Cont.

\begin{tabular}{|c|c|c|c|c|c|c|c|c|}
\hline $\operatorname{Ref}^{*}$ & Preterm/Term & $\begin{array}{l}\text { Phase of } \\
\text { Lactation }\end{array}$ & $\begin{array}{l}\text { Expression } \\
\text { Method }\end{array}$ & Status & $\begin{array}{l}\text { Pre-Pasteurization } \\
\text { Storage }\end{array}$ & $\begin{array}{l}\text { Pasteurization } \\
\text { Equipment }\end{array}$ & Sample Size & Analytical Method ${ }^{\circ}$ \\
\hline \multirow{5}{*}{ [23] } & \multirow{5}{*}{ Term } & \multirow{5}{*}{ Mature } & \multirow{5}{*}{$\begin{array}{c}\text { Hand or } \\
\text { electric/manual } \\
\text { pump. } \\
\text { Occasional drip } \\
\text { milk }\end{array}$} & \multirow{5}{*}{ Frozen } & \multirow{5}{*}{$\begin{array}{l}-20^{\circ} \mathrm{C} \text { up to } \\
15 \text { days; thawing in } \\
\text { a microwave oven }\end{array}$} & \multirow{5}{*}{$\begin{array}{l}62.5^{\circ} \mathrm{C} \text { for } 30^{\prime} ; \\
\text { cooling by } \\
\text { ice-cold water } \\
\text { for } 10^{\prime}\end{array}$} & \multirow{5}{*}{$\begin{array}{l}15 \text { samples from } \\
\text { individual mother or } \\
\text { pool ( } 5 \text { donors) }\end{array}$} & Total fat: crematocrit \\
\hline & & & & & & & & Total protein: Lowry method \\
\hline & & & & & & & & Lactose: picric acid method \\
\hline & & & & & & & & Vitamin A: HPLC \\
\hline & & & & & & & & $\begin{array}{l}\text { Zinc: Atomic absorption } \\
\text { spectrometry }\end{array}$ \\
\hline \multirow[b]{2}{*}{ [24] } & \multirow[b]{2}{*}{ N/A } & \multirow[b]{2}{*}{ Mature } & \multirow[b]{2}{*}{$\mathrm{N} / \mathrm{A}$} & \multirow[b]{2}{*}{ Fresh } & \multirow{2}{*}{$\begin{array}{l}\text { Refrigeration at } 4{ }^{\circ} \mathrm{C} \\
\text { for } 1 \text { to } 2 \text { days; } \\
\text { centrifugation at } \\
2{ }^{\circ} \mathrm{C} \text { for } 1 \mathrm{~h} ;-30^{\circ} \mathrm{C} \\
\text { until testing }\end{array}$} & \multirow[b]{2}{*}{$62.5^{\circ} \mathrm{C}$ for $30^{\prime}$} & \multirow[b]{2}{*}{1 pool-25 donors } & $\begin{array}{l}\text { Immunoglobulins and } \\
\text { lactoferrin: RIA }\end{array}$ \\
\hline & & & & & & & & $\begin{array}{l}\text { Vitamins: labeled cyanocobalamin, } \\
\text { separation of free and } \\
\text { protein-bound vitamins by } \\
\text { gel filtration }\end{array}$ \\
\hline \multirow{3}{*}{ [25] } & \multirow{3}{*}{ N/A } & \multirow{3}{*}{ N/A } & \multirow{3}{*}{$\mathrm{N} / \mathrm{A}$} & \multirow{3}{*}{ Frozen } & \multirow{3}{*}{$\begin{array}{l}-40^{\circ} \mathrm{C} \text { until } \\
\text { analysis }\end{array}$} & \multirow{3}{*}{$62.5^{\circ} \mathrm{C}$ for $30^{\prime}$} & \multirow{3}{*}{1 pool-10 donors } & Total protein: BCA \\
\hline & & & & & & & & Immunoglobulins: ELISA \\
\hline & & & & & & & & $\begin{array}{l}\text { Lysozyme activity: Micrococcus } \\
\text { lysodeikticus turbidimetric assay }\end{array}$ \\
\hline [26] & $\mathrm{N} / \mathrm{A}$ & Mature & N/A & Fresh & Refrigeration & $\begin{array}{l}62.5^{\circ} \mathrm{C} \text { for } 30^{\prime} \text { in } \\
\text { stirred water bath }\end{array}$ & $\begin{array}{l}2 \text { pools }-5 \text { and } 6 \\
\text { donors }\end{array}$ & Immunoglobulins: ELISA \\
\hline [27] & Term & Mature & Electric pump & Fresh & $\begin{array}{l}-80^{\circ} \mathrm{C} \text { until the } \\
\text { analysis }\end{array}$ & $62.5^{\circ} \mathrm{C}$ for $30^{\prime}$ & $\begin{array}{l}10 \text { samples-10 } \\
\text { donors }\end{array}$ & Immunoglobulins: ELISA \\
\hline [28] & $\mathrm{N} / \mathrm{A}$ & Mature & $\mathrm{N} / \mathrm{A}$ & Fresh & No & $63^{\circ} \mathrm{C}$ for $30^{\prime}$ & 23 samples & Immunoglobulins: RIA \\
\hline \multirow{2}{*}{ [29] } & \multirow{2}{*}{ Term } & \multirow{2}{*}{$\begin{array}{l}\text { Colostrum, } \\
\text { transitional } \\
\text { and mature }\end{array}$} & \multirow{2}{*}{$\begin{array}{l}\text { Manual or } \\
\text { pump }\end{array}$} & \multirow[b]{2}{*}{ Fresh } & \multirow{2}{*}{ Refrigeration in ice } & \multirow{2}{*}{$62.5^{\circ} \mathrm{C}$ for $30^{\prime}$} & \multirow{2}{*}{5 samples_-89 donors } & Immunoglobulins: RIA \\
\hline & & & & & & & & Lactoferrin: Laurell method \\
\hline [30] & N/A & $\mathrm{N} / \mathrm{A}$ & Overflow milk & Fresh & $\begin{array}{l}\text { Refrigeration up to } \\
48 \mathrm{~h}\end{array}$ & $62.5^{\circ} \mathrm{C}$ for $30^{\prime}$ & 16 samples & $\begin{array}{l}\text { Electroimmunoassay against } \\
\text { monospecific antiserum }\end{array}$ \\
\hline \multirow{4}{*}{ [31] } & \multirow{4}{*}{ N/A } & & & & & & & Furosine: HPLC \\
\hline & & Colostrum & HMB protocol & Fresh & $\mathrm{N} / \mathrm{A}$ & $62.5^{\circ} \mathrm{C}$ for $30^{\prime}$ & 10 colostrum and & $\begin{array}{l}\text { Carbohydrates: gas } \\
\text { chromatography }\end{array}$ \\
\hline & & & & & & & & Cytokines: ELISA \\
\hline & & & & & & & & Immunoglobulins: ELISA \\
\hline
\end{tabular}


Table 1. Cont.

\begin{tabular}{|c|c|c|c|c|c|c|c|c|}
\hline Ref * & Preterm/Term & $\begin{array}{l}\text { Phase of } \\
\text { Lactation }\end{array}$ & $\begin{array}{l}\text { Expression } \\
\text { Method }\end{array}$ & Status & $\begin{array}{l}\text { Pre-Pasteurization } \\
\text { Storage }\end{array}$ & $\begin{array}{l}\text { Pasteurization } \\
\text { Equipment }\end{array}$ & Sample Size & Analytical Method ${ }^{\circ}$ \\
\hline \multirow{3}{*}{ [32] } & \multirow{3}{*}{$\mathrm{N} / \mathrm{A}$} & \multirow{3}{*}{ Colostrum } & \multirow{3}{*}{$\begin{array}{l}\text { Hand or electric } \\
\text { pump }\end{array}$} & \multirow{3}{*}{ Fresh } & \multirow{3}{*}{$\begin{array}{l}-20{ }^{\circ} \mathrm{C} \text { until } \\
\text { analysis }\end{array}$} & \multirow{3}{*}{$62.5^{\circ} \mathrm{C}$ for $30^{\prime}$} & \multirow{3}{*}{1 pool-11 donors } & Immunoglobulins: ELISA \\
\hline & & & & & & & & $\begin{array}{l}\text { Lysozyme activity: Micrococcus } \\
\text { lysodeikticus turbidimetric assay }\end{array}$ \\
\hline & & & & & & & & $\begin{array}{l}\text { Lactoperoxidase activity: } \\
\text { ABTS assay }\end{array}$ \\
\hline \multirow{3}{*}{ [33] } & \multirow{3}{*}{ Term } & \multirow{3}{*}{ Transitional } & \multirow{3}{*}{ Electric pump } & \multirow{3}{*}{ Fresh } & \multirow{3}{*}{ No } & \multirow{3}{*}{$\begin{array}{l}\text { Metallarredinox: } \\
62.5^{\circ} \mathrm{C} \text { for } 30^{\prime}\end{array}$} & \multirow{3}{*}{1 pool—4 donors } & $\begin{array}{l}\text { IgA, lactoferrin: SDS-PAGE, } \\
\text { Western Blot and mass } \\
\text { spectrometry }\end{array}$ \\
\hline & & & & & & & & $\begin{array}{l}\text { Lipase activity: } \\
\text { p-nitrophenol assay }\end{array}$ \\
\hline & & & & & & & & Available lysine: OPA method \\
\hline [34] & $\mathrm{N} / \mathrm{A}$ & $\mathrm{N} / \mathrm{A}$ & $\mathrm{N} / \mathrm{A}$ & Fresh & N/A & $\begin{array}{l}3 \text { devices: } \\
\text { Sterifeed-Saurin- } \\
\text { Carag: } 62.5^{\circ} \mathrm{C} \\
\text { for } 30^{\prime}\end{array}$ & $\begin{array}{l}10 \text { samples for } \\
\text { Sterifeed; } 6 \text { samples } \\
\text { for Saurin; } 6 \text { samples } \\
\text { for Carag }\end{array}$ & $\begin{array}{l}\text { Lysozyme, sIgA and lactoferrin: } \\
\text { ELISA }\end{array}$ \\
\hline [35] & $\mathrm{N} / \mathrm{A}$ & $\mathrm{N} / \mathrm{A}$ & $\mathrm{N} / \mathrm{A}$ & Frozen & $\begin{array}{l}-20^{\circ} \mathrm{C} \text { until } \\
\text { analysis }\end{array}$ & $62.5^{\circ} \mathrm{C}$ for $30^{\prime}$ & $\begin{array}{l}10 \text { samples-10 } \\
\text { donors }\end{array}$ & $\begin{array}{l}\text { Lysozyme, sIgA and lactoferrin: } \\
\text { ELISA }\end{array}$ \\
\hline \multirow{2}{*}{ [36] } & \multirow[b]{2}{*}{$\mathrm{N} / \mathrm{A}$} & \multirow[b]{2}{*}{$\mathrm{N} / \mathrm{A}$} & \multirow{2}{*}{ Drip milk } & \multirow[b]{2}{*}{ Fresh } & \multirow[b]{2}{*}{ No } & \multirow{2}{*}{$\begin{array}{l}\text { Semi-automated } \\
\text { Holder } \\
\text { pasteurizer }\end{array}$} & \multirow{2}{*}{1 pool-20 donors } & IgA: electroimmunoassay \\
\hline & & & & & & & & $\begin{array}{l}\text { Lysozyme activity: Micrococcus } \\
\text { lysodeikticus turbidimetric assay }\end{array}$ \\
\hline \multirow{4}{*}{ [37] } & \multirow{4}{*}{ N/A } & \multirow{4}{*}{ Mature } & \multirow{4}{*}{ Electric pump } & \multirow{4}{*}{$\begin{array}{l}\text { Frozen and } \\
\text { fresh }\end{array}$} & \multirow{4}{*}{$\begin{array}{l}-70^{\circ} \mathrm{C} \text {; thawing in } \\
\text { cool water }\end{array}$} & \multirow{4}{*}{$\begin{array}{l}62.5^{\circ} \mathrm{C} \text { for } 30^{\prime} \text { in } \\
\text { stirred water bath }\end{array}$} & \multirow{4}{*}{$\begin{array}{l}6 \text { samples: } 3 \text { pools } \\
\text { from HMB and } 3 \\
\text { samples from } 3 \text { donors }\end{array}$} & Total fat: gravimetry \\
\hline & & & & & & & & Fatty acids: gas chromatography \\
\hline & & & & & & & & $\begin{array}{l}\text { Lipase activity: triglyceride } \\
\text { emulsion }\end{array}$ \\
\hline & & & & & & & & Amylase: ELISA \\
\hline \multirow[b]{2}{*}{ [38] } & \multirow[b]{2}{*}{$\mathrm{N} / \mathrm{A}$} & \multirow[b]{2}{*}{ Mature } & \multirow[b]{2}{*}{$\mathrm{N} / \mathrm{A}$} & & $4{ }^{\circ} \mathrm{C}$ overnight; & $\begin{array}{l}\text { Sterifeed: } \\
\text { pre-heated water }\end{array}$ & & $\begin{array}{l}\text { Cytokines and growth factors: } \\
\text { ELISA }\end{array}$ \\
\hline & & & & Frozen & $\begin{array}{l}\text { thawing at } 37^{\circ} \mathrm{C} \\
\text { in water bath, } \\
\text { gently mixed }\end{array}$ & $\begin{array}{l}\text { bath }\left(63.2^{\circ} \mathrm{C}\right) ; \\
62.5^{\circ} \mathrm{C} \text { for } 30^{\prime} ; \\
\text { cooling in cold } \\
\text { water bath }\end{array}$ & $\begin{array}{l}17 \text { pools— } 4 \text { donors } \\
\text { each }\end{array}$ & Fatty acids: gas chromatography \\
\hline
\end{tabular}


Table 1. Cont.

\begin{tabular}{|c|c|c|c|c|c|c|c|c|}
\hline $\operatorname{Ref} *$ & Preterm/Term & $\begin{array}{l}\text { Phase of } \\
\text { Lactation }\end{array}$ & $\begin{array}{l}\text { Expression } \\
\text { Method }\end{array}$ & Status & $\begin{array}{l}\text { Pre-Pasteurization } \\
\text { Storage }\end{array}$ & $\begin{array}{l}\text { Pasteurization } \\
\text { Equipment }\end{array}$ & Sample Size & Analytical Method ${ }^{\circ}$ \\
\hline \multirow{3}{*}{ [39] } & \multirow{3}{*}{$\mathrm{N} / \mathrm{A}$} & \multirow{3}{*}{ Mature } & \multirow{3}{*}{$\mathrm{N} / \mathrm{A}$} & \multirow{3}{*}{ Fresh } & \multirow{3}{*}{$\begin{array}{l}\text { Refrigeration until } \\
\text { analysis }\end{array}$} & \multirow{3}{*}{$\begin{array}{l}62.5^{\circ} \mathrm{C} \text { for } 30^{\prime} \text { in } \\
\text { water bath under } \\
\text { constant agitation }\end{array}$} & \multirow{3}{*}{1 pool—6 donors } & Tocopherol: HPLC \\
\hline & & & & & & & & Fatty acids: gas chromatography \\
\hline & & & & & & & & Cytokines: ELISA \\
\hline [40] & Term & Mature & $\begin{array}{l}\text { Hand or electric } \\
\text { pump }\end{array}$ & Fresh & No & $62.5^{\circ} \mathrm{C}$ for $30^{\prime}$ & 17 samples & $\begin{array}{l}\text { Cytokines and growth factors: } \\
\text { ELISA }\end{array}$ \\
\hline \multirow{2}{*}{ [41] } & \multirow{2}{*}{$\begin{array}{l}\text { Preterm and } \\
\text { Term }\end{array}$} & \multirow{2}{*}{$\begin{array}{l}\text { Transitional } \\
\text { and mature }\end{array}$} & \multirow{2}{*}{$\mathrm{N} / \mathrm{A}$} & \multirow{2}{*}{ Frozen } & \multirow{2}{*}{$\begin{array}{l}-20^{\circ} \mathrm{C} \text { until } \\
\text { analysis }\end{array}$} & \multirow{2}{*}{$\begin{array}{l}\text { LABU-Muttermilch } \\
\text { pasteurizer: } 63^{\circ} \mathrm{C} \\
\text { for } 30^{\prime}\end{array}$} & \multirow{2}{*}{$\begin{array}{l}51 \text { samples-28 } \\
\text { donors }\end{array}$} & IGF and IGFBP: RIA \\
\hline & & & & & & & & EGF: ELISA \\
\hline [42] & Term & Mature & $\mathrm{N} / \mathrm{A}$ & Fresh & No & $62.5^{\circ} \mathrm{C}$ for $30^{\prime}$ & $\begin{array}{l}13 \text { samples-13 } \\
\text { donors }\end{array}$ & Free amino acids: HPLC \\
\hline \multirow{2}{*}{ [43] } & \multirow{2}{*}{$\begin{array}{l}\text { Preterm and } \\
\text { Term }\end{array}$} & \multirow{2}{*}{$\begin{array}{l}\text { Colostrum, } \\
\text { transitional } \\
\text { and mature }\end{array}$} & \multirow{2}{*}{$\mathrm{N} / \mathrm{A}$} & \multirow{2}{*}{ Frozen } & \multirow{2}{*}{ Thawed overnight } & \multirow{2}{*}{$\begin{array}{l}\text { ACE pasteurizer: } \\
62.5^{\circ} \mathrm{C} \text { for } 30^{\prime}\end{array}$} & \multirow{2}{*}{$\begin{array}{l}39 \text { samples-3-4 } \\
\text { donors each }\end{array}$} & Fatty acids: gas chromatography \\
\hline & & & & & & & & $\begin{array}{l}\text { Free amino acids: Amino acid } \\
\text { Analyzer }\end{array}$ \\
\hline [44] & Term & Mature & Manual pump & Frozen & $\begin{array}{l}\text { Refrigeration max } \\
4 \mathrm{~h} ;-20^{\circ} \mathrm{C} \text { up to } \\
3 \text { weeks }\end{array}$ & $62.5^{\circ} \mathrm{C}$ for $30^{\prime}$ & 5 samples—9 pools & Vitamins: HPLC \\
\hline [45] & Term & Mature & Hand or pump & Fresh & Refrigeration & $62.5^{\circ} \mathrm{C}$ for $30^{\prime}$ & 5 each-89 donors & Vitamins: HPLC \\
\hline \multirow{2}{*}{ [46] } & \multirow{2}{*}{$\mathrm{N} / \mathrm{A}$} & \multirow{2}{*}{ Mature } & \multirow{2}{*}{ Electric pump } & \multirow{2}{*}{ Frozen } & \multirow{2}{*}{$\begin{array}{l}-80^{\circ} \mathrm{C} \text { until } \\
\text { analysis }\end{array}$} & \multirow{2}{*}{$62.5^{\circ} \mathrm{C}$ for $30^{\prime}$} & \multirow{2}{*}{$\begin{array}{l}10 \text { samples-10 } \\
\text { donors }\end{array}$} & Vitamin C, Tocopherols: HPLC \\
\hline & & & & & & & & Fatty acids: gas chromatography \\
\hline \multirow{2}{*}{ [47] } & \multirow{2}{*}{$\mathrm{N} / \mathrm{A}$} & Mature & Manual pump & Fresh & No & $62.5^{\circ} \mathrm{C}$ for $30^{\prime}$ & 1 pool-10 donors & Vitamin C, Tocopherols: HPLC \\
\hline & & & & & & & & Fatty acids: gas chromatography \\
\hline [48] & $\mathrm{N} / \mathrm{A}$ & $\mathrm{N} / \mathrm{A}$ & $\mathrm{N} / \mathrm{A}$ & Frozen & Frozen & $63^{\circ} \mathrm{C}$ for $30^{\prime}$ & $\begin{array}{l}50 \text { samples-50 } \\
\text { donors }\end{array}$ & Vitamin A, beta catotene: HPLC \\
\hline [49] & $\mathrm{N} / \mathrm{A}$ & $\begin{array}{l}\text { Colostrum, } \\
\text { transitional } \\
\text { and mature }\end{array}$ & $\mathrm{N} / \mathrm{A}$ & Frozen & $\begin{array}{l}\text { Thawing in ice-filled } \\
\text { plastic container for } \\
15^{\prime}\end{array}$ & $63^{\circ} \mathrm{C}$ for $30^{\prime}$ & 60 samples & Vitamin A: gas chromatography \\
\hline [50] & Preterm and & Transitional & Electric pump & Fresh & No & $62.5^{\circ} \mathrm{C}$ for $30^{\prime}$ & 12 samples-12 & Fat content: gravimetry \\
\hline & & & & & & water & donors & Fatty acids: gas chromatography \\
\hline & & & & & & & & Fatty acids: gas chromatography \\
\hline [b1] & Term & N/A & Hand & Fresh & No & $62.5^{\circ} \mathrm{C}$ for $30^{\prime}$ & 1 pool-16 donors & $\begin{array}{l}\text { L-lactate in milk: enzymatic } \\
\text { biosensor }\end{array}$ \\
\hline
\end{tabular}


Table 1. Cont

\begin{tabular}{|c|c|c|c|c|c|c|c|c|}
\hline $\operatorname{Ref}^{*}$ & Preterm/Term & $\begin{array}{l}\text { Phase of } \\
\text { Lactation }\end{array}$ & $\begin{array}{l}\text { Expression } \\
\text { Method }\end{array}$ & Status & $\begin{array}{l}\text { Pre-Pasteurization } \\
\text { Storage }\end{array}$ & $\begin{array}{l}\text { Pasteurization } \\
\text { Equipment }\end{array}$ & Sample Size & Analytical Method ${ }^{\circ}$ \\
\hline [52] & $\mathrm{N} / \mathrm{A}$ & N/A & $\mathrm{N} / \mathrm{A}$ & Fresh & No & $63^{\circ} \mathrm{C}$ for $30^{\prime}$ & 3 samples-3 donors & $\begin{array}{l}\text { Total fat and fatty acids: gas } \\
\text { chromatography, Infrared } \\
\text { spectroscopy and NMR }\end{array}$ \\
\hline [53] & $\mathrm{N} / \mathrm{A}$ & Transitional & $\mathrm{N} / \mathrm{A}$ & Fresh & No & $\begin{array}{l}62.5^{\circ} \mathrm{C} \text { for } 30^{\prime} ; \\
\text { cooling in running } \\
\text { water }\end{array}$ & 7 samples -1 donor & Fatty acids: gas chromatography \\
\hline [54] & $\mathrm{N} / \mathrm{A}$ & N/A & $\begin{array}{l}\text { Electric/manual } \\
\text { pump }\end{array}$ & Fresh & $\mathrm{N} / \mathrm{A}$ & $\begin{array}{l}62.5^{\circ} \mathrm{C} \text { for } 30^{\prime} ; \\
\text { cooling in stirred } \\
\text { ice-cold water } \\
\text { bath }\end{array}$ & $\begin{array}{l}21 \text { samples-21 } \\
\text { donors }\end{array}$ & Furosine: HPLC \\
\hline [55] & Preterm & $\mathrm{N} / \mathrm{A}$ & Electric pump & Fresh & No & $\begin{array}{l}\text { Sterifeed: } 62.5^{\circ} \mathrm{C} \\
\text { for } 30^{\prime}\end{array}$ & $\begin{array}{l}10 \text { samples-10 } \\
\text { donors }\end{array}$ & Oligosaccharides: HPLC \\
\hline \multirow[t]{2}{*}{ [56] } & Preterm & N/A & Electric pump & Fresh & No & $\begin{array}{l}\text { Sterifeed: } 62.5^{\circ} \mathrm{C} \\
\text { for } 30^{\prime}\end{array}$ & 9 samples-9 donors & Glycosaminoglycans: HPLC \\
\hline & & & & & & & & $\begin{array}{l}\text { Carbohydrates: gas } \\
\text { chromatography }\end{array}$ \\
\hline [57] & $\mathrm{N} / \mathrm{A}$ & Mature & $\mathrm{N} / \mathrm{A}$ & Fresh & Refrigeration & $\begin{array}{l}62.5^{\circ} \mathrm{C} \text { for } 30^{\prime} \text { in } \\
\text { stirred water bath }\end{array}$ & 1 pool—8 donors & $\begin{array}{l}\text { Volatile compounds: gas } \\
\text { chromatography—-mass } \\
\text { spectrometry }\end{array}$ \\
\hline \multirow{3}{*}{ [58] } & \multirow{3}{*}{ Term } & \multirow{3}{*}{ N/A } & \multirow{3}{*}{ Electric pump } & \multirow{3}{*}{ Fresh } & \multirow{3}{*}{ No } & \multirow{3}{*}{$62.5^{\circ} \mathrm{C}$ for $30^{\prime}$} & \multirow{3}{*}{$\begin{array}{l}31 \text { samples-31 } \\
\text { donors }\end{array}$} & MDA and GSH: HPLC \\
\hline & & & & & & & & $\begin{array}{l}\text { GPx activity: Lawrence and Burk } \\
\text { method }\end{array}$ \\
\hline & & & & & & & & ToAC: commercial kit \\
\hline \multirow{5}{*}{ [59] } & \multirow{5}{*}{$\mathrm{N} / \mathrm{A}$} & \multirow{5}{*}{ Mature } & \multirow{5}{*}{ Hand } & \multirow{5}{*}{ Frozen } & \multirow{5}{*}{$\begin{array}{l}-80^{\circ} \mathrm{C} \text { up to } \\
2 \text { weeks }\end{array}$} & \multirow{5}{*}{$62.5^{\circ} \mathrm{C}$ for $30^{\prime}$} & \multirow{5}{*}{$\begin{array}{l}30 \text { samples-10 } \\
\text { donors }\end{array}$} & Total fat : creamatocrit \\
\hline & & & & & & & & $\begin{array}{l}\text { Fatty acids and volatiles: gas } \\
\text { chromatography }\end{array}$ \\
\hline & & & & & & & & MDA: TBARS \\
\hline & & & & & & & & $\begin{array}{l}\text { Tocopherols and ascorbic acid: } \\
\text { HPLC }\end{array}$ \\
\hline & & & & & & & & ToAC: ORAC \\
\hline [60] & $\mathrm{N} / \mathrm{A}$ & Mature & Hand & Frozen & $\begin{array}{l}-40^{\circ} \mathrm{C} \text { in } \mathrm{HMB} \\
-18^{\circ} \mathrm{C} \text { in lab }\end{array}$ & $62.5^{\circ} \mathrm{C}$ for $30^{\prime}$ & 1 pool—5 donors & $\begin{array}{l}\text { Free nucleotide monophosphates: } \\
\text { capillary electrophoresis_-mass } \\
\text { spectrometry }\end{array}$ \\
\hline
\end{tabular}


Table 2. DM components not affected by Holder Pasteurization (HoP) (consensus on results).

\begin{tabular}{|c|c|}
\hline Components & Reference \\
\hline Total Nitrogen Content & [18] \\
\hline \multicolumn{2}{|l|}{ Cytokines } \\
\hline IL2, IL4, IL5, IL13 & {$[31,38]$} \\
\hline IL12p70 & {$[31,38,39]$} \\
\hline IL17 & {$[31,39]$} \\
\hline \multicolumn{2}{|l|}{ Growth Factors } \\
\hline EGF & {$[40,41]$} \\
\hline TGF $\beta 1$ & [40] \\
\hline TGF $\beta 2$ & [31] \\
\hline MCP-1 & [31] \\
\hline \multicolumn{2}{|l|}{ Amino acids } \\
\hline Free amino acids & [43] \\
\hline Taurine, methionine, cystine, glutamate & {$[42,43]$} \\
\hline \multicolumn{2}{|l|}{ Vitamins } \\
\hline D, E, B2 & [44] \\
\hline B5, Biotin, B3 & {$[45]$} \\
\hline B12 & {$[21,24,44,45]$} \\
\hline$\underline{\text { Zinc }}$ & [23] \\
\hline \multicolumn{2}{|l|}{ Lipids } \\
\hline \multicolumn{2}{|l|}{ Polyunsaturated fatty acid $n 3$} \\
\hline $20: 5$ & {$[37,50]$} \\
\hline 22:5 & {$[38,47,50]$} \\
\hline 22:6 & {$[37,39,43,47,50]$} \\
\hline \multicolumn{2}{|l|}{ Polyunsaturated fatty acid $n 6$} \\
\hline $18: 2$ & {$[37-39,43,47,50-53]$} \\
\hline $18: 3$ & {$[39,43,47,50]$} \\
\hline $20: 2$ & {$[47,50]$} \\
\hline 20:3 & {$[39,47,50]$} \\
\hline $20: 4$ & {$[37-39,43,47]$} \\
\hline $22: 4$ & {$[37,47,50]$} \\
\hline $22: 5$ & {$[38,47]$} \\
\hline \multicolumn{2}{|l|}{ Monounsaturated fatty acid } \\
\hline $14: 1$ & {$[37,39,43,47,50]$} \\
\hline $15: 1$ & {$[47,50,52]$} \\
\hline $16: 1$ & {$[37-39,43,47,50-53]$} \\
\hline $17: 1 ; 22: 1$ & {$[47,50]$} \\
\hline 20:1 & {$[39,47,50]$} \\
\hline $24: 1$ & {$[37,47,50]$} \\
\hline \multicolumn{2}{|l|}{ Saturated fatty acid } \\
\hline $10: 0,16: 0$ & {$[37-39,43,47,50-52]$} \\
\hline 15:0 & {$[39,47,50,51]$} \\
\hline $17: 0$ & {$[39,47,50]$} \\
\hline 20:0 & {$[39,47,50,52]$} \\
\hline 21:0 & [47] \\
\hline 22:0 & {$[47,50]$} \\
\hline 24:0 & {$[37,50]$} \\
\hline \multicolumn{2}{|l|}{ Saccharides } \\
\hline Oligosaccharides & [55] \\
\hline Glycosaminoglycans & [56] \\
\hline Myoinositol & {$[31,54]$} \\
\hline Lactose & {$[18,19,23,31,54]$} \\
\hline \multicolumn{2}{|l|}{ Oxidative Stress Markers } \\
\hline Malondialdehyde & {$[58,59]$} \\
\hline ORAC and Hexanal & [59] \\
\hline
\end{tabular}


Table 3. DM components affected significantly by HoP (consensus on results).

\begin{tabular}{|c|c|c|}
\hline Components & Effect of HoP & References \\
\hline \multicolumn{3}{|l|}{ Immunoglobulins } \\
\hline IgG4 & Reduction (\% not reported) & {$[31]$} \\
\hline \multicolumn{3}{|l|}{ Enzymes } \\
\hline Lipase & Complete loss & {$[21,33,37]$} \\
\hline Alkaline phosphatase & Complete loss & [21] \\
\hline Amylase & Reduction (15\%) & [37] \\
\hline \multicolumn{3}{|l|}{ Cytokines } \\
\hline IL7 & Increase (\% not reported) & [31] \\
\hline MIP-1 $\beta$ & Reduction (\% not reported) & [31] \\
\hline MCAF/MCP-1 & Reduction (\% not reported) & [39] \\
\hline \multicolumn{3}{|l|}{ Growth Factors } \\
\hline IGF1, IGF2, IGFBP2, IGFBP3 & Reduction (\% not reported) & [41] \\
\hline EPO & Reduction (\% not reported) & [40] \\
\hline HB-EGF, HGF & Reduction (\% not reported) & [38] \\
\hline GM-CSF & Increase (\% not reported) & [31] \\
\hline \multicolumn{3}{|l|}{ Hormones } \\
\hline Insulin, Adiponectin & Reduction (\% not reported) & [17] \\
\hline \multicolumn{3}{|l|}{ Free amino acids } \\
\hline Arginine, leucine & Increase & {$[43]$} \\
\hline Aspartate & Reduction (\% not reported) & [43] \\
\hline Glutamine & Increase (\% not reported) & [42] \\
\hline \multicolumn{3}{|l|}{ Vitamins } \\
\hline Ascorbic + Dehydroascorbic & Reduction $(12 \%)$ & [47] \\
\hline Ascorbic Acid & Reduction $(16.2 \%-26 \%)$ & {$[46,47]$} \\
\hline B6 & Reduction (15\%) & {$[44,46]$} \\
\hline \multicolumn{3}{|l|}{ Oxidative Stress Markers } \\
\hline $\begin{array}{l}\text { Glutathione, Glutathione peroxidase } \\
\text { activity, Total antioxidant capacity }\end{array}$ & Reduction (\% not reported) & [58] \\
\hline Lactulose & $\begin{array}{l}\text { Increase (\% not reported)/(Not } \\
\text { detected in all samples) }\end{array}$ & {$[31,54]$} \\
\hline Nucleotide monophosphate content & Increase (\% not reported) & {$[60]$} \\
\hline
\end{tabular}

Table 4. DM components affected by HoP (discordant results).

\begin{tabular}{|c|c|c|}
\hline Components & Effects of HoP & References \\
\hline Total Protein Content & Reduction (\% not reported) & $\begin{array}{c}\text { Significant: }[17,19,20] \\
\text { Not significant: }[21-23,37]\end{array}$ \\
\hline \multicolumn{3}{|l|}{ Immunoglobulins } \\
\hline $\operatorname{Ig} \mathrm{A}$ & Reduction $(20 \%-62 \%)$ & $\begin{array}{c}\text { Significant: }[25,28,29,31,32] \\
\text { Not significant: }[20,24,26,27,30,35]\end{array}$ \\
\hline sIgA & Reduction (20\%-50\%) & $\begin{array}{c}\text { Significant: }[35] \\
\text { Not significant: }[21,34]\end{array}$ \\
\hline $\operatorname{IgM}$ & Reduction (50\%-100\%) & $\begin{array}{c}\text { Significant: }[28,29,31,32] \\
\text { Not significant: }[20,24,26]\end{array}$ \\
\hline $\operatorname{IgG}$ & Reduction (23\%-100\%) & $\begin{array}{c}\text { Significant: [32] } \\
\text { Not significant: }[20,24,26,28-30]\end{array}$ \\
\hline
\end{tabular}


Table 4. Cont.

\begin{tabular}{|c|c|c|}
\hline Components & Effects of HoP & References \\
\hline Lactoferrin & Reduction (35\%-90\%) & $\begin{array}{c}\text { Significant: }[35] \\
\text { Not significant: }[24,29,30,33,34]\end{array}$ \\
\hline \multicolumn{3}{|l|}{ Lysozyme } \\
\hline concentration & Reduction $(20 \%-69 \%)$ & $\begin{array}{l}\text { Significant: [34] (Sterifeed and Carag), [35] } \\
\text { Not significant: }[20,21,30,34] \text { (Saurin) }\end{array}$ \\
\hline activity & Reduction (\% not reported) & $\begin{array}{l}\text { Significant: }[25,32] \\
\text { Not significant: }[24]\end{array}$ \\
\hline \multicolumn{3}{|l|}{ Cytokines } \\
\hline IL1beta, IL6 & Reduction (\% not reported) & $\begin{array}{l}\text { Significant: [39] } \\
\text { Not significant: [31] }\end{array}$ \\
\hline IL8 & Increase (\% not reported) & $\begin{array}{l}\text { Significant: [38-40] } \\
\text { Not significant [31] }\end{array}$ \\
\hline IL10 & Reduction (\% not reported) & $\begin{array}{l}\text { Significant: }[38,39] \\
\text { Not significant: }[31]\end{array}$ \\
\hline TNF alfa & Reduction (\% not reported) & $\begin{array}{l}\text { Significant: }[38,39] \\
\text { Not significant: }[31]\end{array}$ \\
\hline INF gamma & Reduction (\% not reported) & $\begin{array}{c}\text { Significant: [38] } \\
\text { Not significant: }[31,39]\end{array}$ \\
\hline \multicolumn{3}{|l|}{ Vitamins } \\
\hline A & $\begin{array}{c}\text { Increase (\% not reported)/reduction } \\
(34 \%)\end{array}$ & $\begin{array}{c}\text { Significant: }[49] \\
\text { Not significant: }[23,44,48]\end{array}$ \\
\hline Folacin & Reduction $(10 \%-30 \%)$ & $\begin{array}{c}\text { Significant: }[44] \\
\text { Not significant: }[21,24,29]\end{array}$ \\
\hline $\mathrm{C}$ & Reduction $(19.9 \%-36 \%)$ & $\begin{array}{l}\text { Significant: }[44,46] \\
\text { Not significant: }[29]\end{array}$ \\
\hline alfa- and gamma-Tocopherol & Reduction (12\%-47\%) & $\begin{array}{l}\text { Significant: }[39,47] \\
\text { Not significant: }[46]\end{array}$ \\
\hline delta-Tocopherol & Reduction (\% not reported) & $\begin{array}{c}\text { Significant: [39] } \\
\text { Not significant: [46] }\end{array}$ \\
\hline Total fat content & $\begin{array}{c}\text { Reduction (\% not reported)/Increase } \\
\text { (\% not reported) }\end{array}$ & $\begin{array}{c}\text { Significant: }[17-19] \\
\text { Not significant: }[23,37,50,51]\end{array}$ \\
\hline \multicolumn{3}{|l|}{ Polyunsaturated fatty acid $n 3$} \\
\hline $18: 3$ & Reduction (\% not reported) & $\begin{array}{c}\text { Significant: }[53] \\
\text { Not significant: }[38,39,47,50]\end{array}$ \\
\hline \multicolumn{3}{|l|}{ Monounsaturated fatty acid } \\
\hline $18: 1$ & Increase/reduction (\% not reported) & $\begin{array}{c}\text { Significant: }[38] \\
\text { Not significant: }[39,47,50,53]\end{array}$ \\
\hline \multicolumn{3}{|l|}{ Saturated fatty acid } \\
\hline $14: 0$ & Increase/reduction (\% not reported) & $\begin{array}{c}\text { Significant: [38] } \\
\text { Not significant: }[37,39,43,47,50,52,53]\end{array}$ \\
\hline $12: 0$ & Increase/reduction (\% not reported) & $\begin{array}{c}\text { Significant: }[38,43] \\
\text { Not significant: }[37,39,47,50-52] \\
\end{array}$ \\
\hline $18: 0$ & Increase/reduction (\% not reported) & $\begin{array}{c}\text { Significant: }[38] \\
\text { Not significant: }(2015)[37,39,43,47,50-52]\end{array}$ \\
\hline Glucose & Reduction/Increase (\% not reported) & $\begin{array}{c}\text { Significant: [17] (increase), [54] (reduction) } \\
\text { Not significant: [31] }\end{array}$ \\
\hline
\end{tabular}

\subsection{Energy Content}

The first question that needs to be answered concerning the nutrition of all infants with pasteurized DM is: is this milk as nutritive as fresh HM? Only two studies have been found to have assessed the effects of HoP on the energy content of milk, with conflicting results. Ley et al. [17] reported that the 
HM energy content was not modified by pasteurization, whereas García-Lara et al. [18] found that the energy content was decreased significantly. The two studies used different approaches to calculate the energy content; in the first case [17], a direct measurement was performed by means of bomb calorimetry, whereas in the second study the energy content was calculated on the basis of the fat, protein and lactose contents, as measured by means of infrared spectroscopy [18]. The discrepancy in the results may be due to statistical artifacts rather than to real differences, since the percent decrease measured in the two studies was similar.

Overall, current evidence does not support the hypothesis of a relevant change in energy intake by feeding the infant with pasteurized rather than raw DM.

\subsection{Nitrogenous Compounds}

\subsubsection{Protein Content}

The composition of the HM protein fraction varies from mother to mother, and changes during lactation. The protein content of term milk is estimated to be approximately 0.9 to $1.2 \mathrm{~g} / \mathrm{dL}$, while this value is higher for preterm milk [61]. The true protein content of HM is often overestimated, due to its high proportion of non-protein nitrogen [62]. In a study by Vieirà et al. [19], the average DM protein concentration, as assessed by means of an infrared analyzer, was significantly reduced by the HoP treatment, as was also found for colostrum [20]. Other studies, on the contrary, did not observe any significant change in protein content $[17,21-23]$, even when the total nitrogen content was measured indirectly [18].

In conclusion, the majority of the examined reports indicates that HoP does not affect the protein content of DM. A statistically significant reduction was only found in two studies, although one of the studies, involving mature milk [19], reported a very slight reduction $(-3.9 \%)$, similar to that found in the studies that claimed no effect on total protein content.

\subsubsection{Immunoglobulins (Igs)}

The effect of HoP on the DM concentration of different classes of immunoglobulins has been investigated in several studies, the first being published in 1977 by Ford and colleagues [24].

IgAs and secretory IgAs (sIgAs) are the most extensively investigated classes, and almost all the published studies report a reduction following HoP. A significant reduction in IgAs following HoP was measured by means of Enzyme-Linked Immunosorbent Assays (ELISA) [25-27] and by means of Radial Immunodiffusion Assays (RIA) [28,29]. An IgA reduction was also reported in older studies [24,30], although no statistical significance was reached, perhaps due to weak experimental design. Ford et al. [24] analyzed a single pooled milk sample, while Evans et al. [30] used samples derived from overflow milk. The detrimental effect of $\mathrm{HoP}$ on IgAs was also confirmed on colostrum samples [20,31,32]. A reduction in protein bands recognized by means of anti-IgAs antibodies, although not quantified, was also reported [33]. Secretory IgAs, the dimeric forms of IgAs, were also found to have decreased following the pasteurization of DM [21,34,35], although not always significantly.

The other Ig classes were investigated in a smaller number of studies, whose results are partially contrasting, due to the extremely low Ig concentrations, and subsequent difficult detectability in the milk samples. However, the majority of the studies found some degree of reduction.

IgM concentrations were measured in mature DM after pasteurization, and were found to be significantly decreased [26,28,29], or even completely degraded [24]. The low resistance of IgMs to pasteurization has also been confirmed more recently on colostrum [20,31,32]. The same behavior has also been observed for IgG concentrations in both milk [26,28-30] and in colostrum [20,31,32]. The specific thermal resistance of different IgGs subclasses was also detailed: IgG1 were not affected by HoP, while IgG4 were reduced, and IgG2 and IgG3 were undetectable in both fresh and pasteurized milk samples [31]. 
As an overall conclusion, the results from the previous reports clearly indicate that one of the main detrimental effects of HoP is a reduction in all classes of immunoglobulins, probably due to the complex structure of these molecules.

\subsubsection{Lactoferrin and Lysozyme}

The immunoprotective protein constituents of $\mathrm{HM}$, with bacteriostatic properties, include lysozyme and lactoferrin [62].

Lactoferrin is an iron-binding protein that reduces the availability of the free iron required by iron-dependent pathogens, and therefore is able to inhibit their growth. Moreover, it can disrupt the bacterial cell membrane by binding to the lipid-A portion of lipopolysaccharides on the bacterial cell surface [63]. Lactoferrin has been investigated in several studies, using different techniques (ELISA, RIA, monospecific antisera) [24,29-32], all of which report a reduction in its concentration, with a percentage ranging from $35 \%$ to $90 \%$, but the reduction was only reported as significant by Christen et al. [35]. Additionally, a reduction in the lactoferrin-containing band, by means of a protein electrophoresis semi-quantitative method, was reported [33]. Since the bactericidal activity of lactoferrin is maintained by bactericidal peptides that form during its digestion, it is possible that part of the activity is still retained in pasteurized HM despite reduction of the protein [62]. One recent survey [64] conducted by means of non-reducing protein electrophoresis, has reported that lactoferrin aggregation, rather than degradation, occurs following DM pasteurization with HoP. Whether this aggregation causes a decrease in lactoferrin bactericidal activity, is still not known.

A similar pattern emerges for lysozyme, whose concentration has been found to be reduced after pasteurization in several studies, with a percentage ranging from $20 \%$ to $85 \%$. The biological activity of lysozyme was tested by Ford et al. [24], who did not find any significant difference. A significant reduction in lysozyme activity after $\mathrm{HoP}$ was found by other authors [25,36], even in colostrum [32]. It should be pointed out that, in those works, the lysozyme activity was always determined using a Micrococcus lysodeikticus-based turbidimetric assay, which measures to what extent bacterial growth is prevented by the addition of lysozyme-containing samples. Since DM is a complex mixture of several anti-bacterial enzymes and factors, it is not possible to discriminate if the reduction is due only to a decrease in lysozyme concentration.

\subsubsection{Other Enzymes}

Owing to their different responses to heating, enzymes and their activity are commonly considered as markers for assessment of thermal treatments. Some of these enzymes (such as lactoperoxidase and alkaline phosphatase) are considered technological markers for pasteurization in bovine milk. Data on lactoperoxidase are scarce for $\mathrm{HM}$, since its concentration is below the detection limit of commercial kits [24,32]. Alkaline phosphatase has also been found to be completely inactivated by HoP [21], as commonly found in bovine milk.

Because of the compensatory function of several HM enzymes for nutrient digestion in newborns [65], some researchers have focused on the activity of lipase and amylase milk enzymes. A complete degradation (both in concentration and enzymatic activity) has been found for lipoprotein lipase and bile salt dependent lipase [21,33,37], while amylase activity was partly retained [37]. The clinical relevance of variations in the enzymatic activity of these proteins still has to be investigated. In particular, the hypothesis of a reduction in nutrient absorption through feeding of pasteurized rather than raw HM, especially as far as lipid digestion is concerned, cannot be ruled out.

\subsubsection{Cytokines}

Although cytokines are immunomodulatory components, it appears that most of those found in HM are anti-inflammatory, thereby possibly lessening the effect of infections [62]. The effect of pasteurization on several cytokines has been evaluated in colostrum [31]. Interleukin (IL)1 $\beta$, IL2, IL4, IL5, IL6, IL8, IL10, IL12, IL13, IL17, Interferon (IFN)- $\gamma$, the Tumor Necrosis Factor 
(TNF)- $\alpha$ and Monocyte Chemotactic Protein (MCP)-1 were not significantly affected by the process. Rather remarkably, IL7 increased significantly after pasteurization, perhaps due to its release from cellular and/or fat compartments into the aqueous fraction, while Macrophage Inflammatory Protein-1 $\beta$ (MIP-1 $\beta$ ) was significantly reduced [31]. IL2, IL4, IL5, IL12 and IL13 were unaffected even in pasteurized mature DM [38], while IL10 was decreased [38-40], as were IL1 $\beta$ [38], IFN- $\gamma$ [39], IL6 [39] and TNF- $\alpha$ [38,39]. A significant increase in IL8, following HoP in mature DM, was also found $[38,39]$.

In short, different degrees of thermal resistance were found for different cytokines, and the biological relevance of this altered balance in specific situations still remains to be addressed.

\subsubsection{Growth Factors}

To date, only a few studies have evaluated the variation of growth factors (GF) in human milk after pasteurization, and each one has focused on one specific GF.

Transforming GF (TGF)- $\beta 2$ was found to be stable in colostrum [31]. Epidermal GF (EGF) [40,41] and TGF- $\beta 1$ [40] showed no difference before and after HoP. Heparin-Binding Epidermal-like GF (HB-EGF) was unaffected by heating, whereas Hepatocyte GF (HGF) was reduced to a great extent [38]. Insulin-like GF (IGF)-1 and 2, as well as IGF binding proteins 2 and 3, were reduced to a variable extent by pasteurization [41]. Granulocyte-Macrophage Colony-Stimulating Factor (GM-CSF) concentrations increased significantly after pasteurization, while the Granulocyte-Colony Stimulating Factor (GCSF) was not detected in any sample, before or after HoP, either in colostrum [31], or in mature milk [38].

Once again, as in the case of cytokines, the low number of studies to date, and the relevant variability between different growth factors, does not allow any conclusion on the effect of HoP to be generalized.

\subsubsection{Amino Acids}

The DM amino acid composition was investigated in two studies, one [42] focusing only on sulfur amino acids (cysteine and methionine), and a few free amino acids. The authors reported that no significant modification was caused by HoP, with the exception of an increase in free glutamine [42]. In another study [43], the amino acid profile of pre- and post-pasteurization DM was found to be significantly different for arginine and leucine, to increase following HoP, and to decrease for aspartate. Nevertheless, the biological relevance of these variations was reported to be low, since the differences, although significant, were small.

The available lysine content, which is considered a nutritional marker, as lysine residues are targeted by proteases during digestion, was evaluated in two studies, with discordant results: Silvestre et al. [22] showed a 30\% reduction (statistically significant) using a fluorimetric method, while Baro et al. [33] observed an increase in the concentration of this amino acid, measured by means of $o$-phthaldialdehyde. In this case, the discordant results may partially be due to the different experimental designs of the two studies, one of which simulated HoP [22], and the other used a commercial Holder pasteurizer [33].

\subsection{Hormones}

Insulin, adiponectin [17] and erythropoietin [40] concentrations were all reported to be decreased significantly after HoP, although the paucity of the reports does not allow any conclusion on the issue to be generalized.

\subsection{Vitamins}

The evidence on the effect of HoP on HM vitamins is contrasting. Folacin and vitamin B12 were evaluated in different studies with conflicting results, probably due to the significant instability of vitamins and to the variability between the study sampling and analysis methods of the studies. Ford et al. [24] found an important reduction in the capacity of DM to bind added vitamins after 
HoP, as measured by adding excess cyanocobalamin (vitamin B12 analog), while other studies [21,44] did not find any significant difference for vitamin B12 analyzed by means of a chemiluminescence immunoassay and a competitive protein binding assay. Stability of riboflavin, biotin, and the total pantothenic acid contents (vitamin B5) has also been observed [45]. A non-significant decrease was found for folacin (vitamin B9) after HoP [21,24,45]. Van Zoeren-Grobben et al. on the other hand, found a $30 \%$ reduction in folacin as a consequence of $\mathrm{HoP}$, and a significant $15 \%$ reduction in vitamin B6 [44].

While the vitamin C concentration was unambiguously reported to be reduced by HoP [44,46,47], fat-soluble vitamins showed different responses to pasteurization. Vitamin D was unaffected [44]. In some reports, vitamin A was found to be reduced [48,49], but other authors found it stable [23,44], while tocopherols (vitamin E) were found not to be affected in [44,46], but reduced in $[39,47]$.

To summarize, the available data seem to confirm a higher heat sensitivity for water soluble vitamins, and in particular for vitamin $C$, which is known to be highly susceptible to several technological treatments, including freezing and refrigeration [66], while most of the studies seem to indicate a higher retention of fat soluble vitamins (A, D, and partially E).

\subsection{Zinc}

Zinc levels have not resulted not to be significantly affected by HoP; however, a variation in the distribution of zinc was observed, with a significant increase in the fat fraction and a decrease in whey, possibly as a result of the denaturation of zinc binding proteins, thereby indicating possible consequences on zinc bioavailability to the infant [23].

\subsection{Lipids}

The total lipid content was evaluated in several studies: a significant reduction was found following HoP, using infrared analyzers [17-19], which, however, do not directly measure the fat content. Other authors have not found significant differences, when using different analysis techniques [23,37,50,51]. The total fatty acid profile was always found to be unaffected by pasteurization $[37,39,43,47,50,52]$, with the exception of one study, which reported slight changes in the relative composition of medium-chain fatty acids (MCFAs) [38], and one of which reported a small decrease in 18:3 fatty acid [53]. Lepri et al. [51] found a more than two-fold free fatty acid content increase after pasteurization. A potential increase in the DM free fatty acid fraction following HoP, provided it does not cause off-flavors, may not be undesirable, as increased free fatty acids are known to be more readily absorbed in the digestive system, thus resulting in a possibly increased nutritive potential.

\subsection{Saccharides}

Several studies have evaluated the effect of HoP on lactose in mature milk and colostrum. In all cases, and using different analytical techniques, no significant difference was found before and after HoP $[18,19,23,31,54]$. The myoinositol levels were not affected by HoP either [31,54], while glucose was found to be increased [17], stable [31] and reduced [54], although all of the reported variations were low.

Glycosaminoglycans and oligosaccharides have recently been investigated in preterm milk, and no variation after the pasteurization process was observed [55,56]. These human milk glycans have been demonstrated to influence the health of newborns, since they possess specific biological properties, such as an anti-infective role, anti-oxidant functions and prebiotic effects [67].

In short, the evidence to date points toward the stability of DM saccharides during pasteurization by HoP, both as free molecules and as part of biologically active compounds, such as glycosaminoglycans and oligosaccharides. 


\subsection{Indicators of Thermal Treatment}

Lactulose, a disaccharide formed through the isomerization of lactose, and furosine, an intermediate of the Maillard reaction, have been searched for in DM, since they are used in the food industry to differentiate between different kinds of heat-treated bovine milk, depending on the intensity of the heat treatment applied. Since HM has more than double the lactose content than bovine milk, lactulose formation has been found to be triggered more by pasteurization than is normally found for pasteurized bovine milk, in both milk [67] and colostrum [31]. Furosine was only found to have formed following pasteurization in colostrum samples [31], while it was not found in any milk sample [67].

Contador et al. [57] have very recently published a detailed analysis of volatile compounds in fresh and pasteurized HM, which supports the idea of some thermal-induced modification during HoP. The preservation of the original volatile compounds in HM is important, since they can negatively affect its quality, and indicate that undesired reactions have taken place during the treatment (e.g., lipid oxidation, Maillard reaction, etc.). A significant change in many volatiles was detected after HoP in DM; some of these volatiles (aldehydes, furans and pyrans) are undesired, and considered an index of thermal degradation.

\subsection{Oxidative Stress Markers}

The oxidative balance of raw and pasteurized HM was assessed by measuring both the accumulation of oxidants and the activity of oxidant scavengers. Malondialdehyde concentrations were not found to be significantly affected by HoP but, on the other hand, glutathione concentrations, glutathione peroxidase activity and Total Antioxidant Capacity were significantly reduced, thus indicating a decrease in the oxidative scavenging capacity of HM [58]. In a more recent assay [59], no change in malondialdehyde concentration, total antioxidant capacity (as measured by means of Oxygen Radical Absorbance Capacity assay), or hexanal concentration has been found, thus indicating no lipid oxidation and no decrease in oxidant scavengers.

\subsection{Organic Acids}

Only one study has assessed the L-lactate content of HM, following HoP, using a biosensor based on an immobilized lactate oxidase enzyme. The results showed a significant increase, probably due to the release from interaction with other substances [51].

\subsection{Recently Published Research}

One study has recently highlighted an increase in the nucleotide monophosphate content following pasteurization, measured by means of mass spectrometry. These compounds are considered as immune-enhancers and seem to play a role as sleep-inducers, so their increase in HM may be a positive consequence of HoP [60]. Nevertheless, further evidence is needed before any claim on the issue could be formulated.

\section{Discussion and Conclusions}

The present review shows a significant variability in the data reported in the scientific literature concerning the effects of HoP on the biological components of HM.

A possible explanation for this variability may be the heterogeneity of the test protocols applied in the studies (e.g., in terms of sample origin, storage conditions or analysis methods). Another important source of variability is represented by the fact that the Holder pasteurization of donor milk is often simulated on small aliquots, rather than being performed following HMB-implemented protocols. Moreover, modern pasteurizers require significantly less time for heating and cooling than older ones, thus changing the kinetics of the thermal response for heat-sensible compounds. Additionally, it 
appears that some biochemical patterns were investigated more extensively than others, while some other milk components were not considered at all.

The results of the review can be summarized as follows. Saccharides are not significantly affected by the heat treatment, as either free molecules or as part of biologically active compounds. The total lipid content is preserved by HoP, as is its fatty acid composition. This finding is of paramount importance since it suggests that pasteurization is able to preserve both the nutritional and biological properties relevant to the development of the central nervous system associated with some of these fatty acids. Consistently, fat soluble vitamins also seem to be unaffected, while water soluble vitamins, and vitamin $C$ in particular, are generally reported as significantly decreased. The results concerning specific biologically active molecules (such as cytokines and growth factors) remain uncertain, due to the vast number of different compounds analyzed in each study, and to the paucity of comparable results.

Proteins are more significantly affected by HoP. In fact, specific proteins with significant immunologic and anti-infective action (such as immunoglobulins and lactoferrin) are reduced by pasteurization. A substantial reduction in the enzymatic activity has also been observed. The review thus confirms the main concerns about Holder pasteurization of HM, and the need for future strategies to prevent and/or limit DM protein degradation. The available data confirm that HoP affects several HM components to variable degrees, even though it is rather difficult to quantify the degradation level. Nonetheless, clinical practices demonstrate that many beneficial properties of human milk remain, even after pasteurization.

Future studies should be aimed at confirming the currently available data by investigating more reproducible analytic settings, while avoiding the introduction of potential biases, in order to understand the real effects of pasteurization on mother's milk. Moreover, further studies should be focused on new pasteurization techniques in order to improve the biological quality and safety of DM.

Author Contributions: Chiara Peila has made a substantial contribution to the conception, design and drafting of the manuscript. Alessandra Coscia has been involved in drafting the manuscript and revising it critically for its important intellectual content. Enrico Bertino and Francesco Cresi revised the manuscript critically for its important intellectual content and gave the final approval of the version to be published. Guido Moro, Marzia Giribaldi and Laura Cavallarin participated in the design of the study, were involved in the drafting of the manuscript, and revised it critically for its important intellectual content. All of the authors read and approved the final version of the paper.

Conflicts of Interest: The authors declare no conflict of interest.

\section{References}

1. American Academy of Paediatrics. Breastfeeding and use of human milk. Pediatrics 2012, 129, e827-e841.

2. Hamosh, M. Protective function of proteins and lipids in human milk. Biol. Neonate 1998, 74, $163-176$. [CrossRef] [PubMed]

3. Horta, B.L.; Victora, C.G.; World Health Organization. Long-Term Effects of Breastfeeding: A Systematic Review; WHO Library: Geneva, Switzerland, 2013.

4. Newman, J. How breast milk protects newborns. Sci. Am. 1995, 273, 76-79. [CrossRef] [PubMed]

5. Italian Association of Human Milk Banks; Arslanoglu, S.; Bertino, E.; Tonetto, P.; De Nisi, G.; Ambruzzi, A.M.; Biasini, A.; Profeti, C.; Spreghini, M.R.; Moro, G.E. Guidelines for the establishment and operation of a donor human milk bank. J. Matern. Fetal Neonat. Med. 2010, 23, 1-20.

6. Human Milk Banking Association of North America. Guidelines for the Establishment and Operation of a Donor Human Milk Bank; Human Milk Banking Association of North America: Raleigh, NC, USA, 2000.

7. Boyd, C.A.; Quigley, M.A.; Brocklehurst, P. Donor breast milk versus infant formula for preterm infants: Systematic review and meta-analysis. Arch. Dis. Child. Fetal Neonat. Ed. 2007, 92, F169-F175. [CrossRef] [PubMed]

8. McGuire, W.; Anthony, M.Y. Donor human milk versus formula for preventing necrotising enterocolitis in preterm infants: Systematic review. Arch. Dis. Child. Fetal Neonat. Ed. 2003, 88, F11-F14. [CrossRef]

9. Quigley, M.A.; Henderson, G.; Anthony, M.Y.; McGuire, W. Formula milk versus donor breast milk for feeding preterm or low birth weight infants. Cochrane Database Syst. Rev. 2007, 4. [CrossRef] 
10. Rønnestad, A.; Abrahamsen, T.G.; Medbø, S.; Reigstad, H.; Lossius, K.; Kaaresen, P.I.; Egeland, T.; Engelund, I.E.; Irgens, L.M.; Markestad, T. Late onset septicemia in a Norwegian national cohort of extremely premature infants receiving very early full human milk feeding. Pediatrics 2005, 115, 269-276. [CrossRef] [PubMed]

11. Schanler, R.J.; Lau, C.; Hurst, N.M.; Smith, E.O. Randomized trial of donor human milk versus preterm formula as substitutes for mothers' own milk in the feeding of extremely premature infants. Pediatrics 2005, 116, 400-406. [CrossRef] [PubMed]

12. Tully, D.B.; Jones, F.; Tully, M.R. Donor milk: What's in it and what's not. J. Hum. Lact. 2001, 17, $152-155$. [CrossRef] [PubMed]

13. Ogundele, M.O. Techniques for the storage of human breast milk: Implications for anti-microbial functions and safety of stored milk. Eur. J. Pediatr. 2000, 159, 793-797. [CrossRef] [PubMed]

14. ESPGHAN Committee on Nutrition; Arslanoglu, S.; Corpeleijn, W.; Moro, G.; Braegger, C.; Campoy, C.; Colomb, V.; Decsi, T.; Domellöf, M.; Fewtrell, M.; et al. Donor human milk for preterm infants: Current evidence and research directions. J. Pediatr. Gastroenterol. Nutr. 2013, 57, 535-542.

15. Björksten, B.; Burman, L.G.; De Château, P.; Fredrikzon, B.; Gothefors, L.; Hernell, O. Collecting and banking human milk: To heat or not to heat. Br. Med. J. 1980, 281, 765-769. [CrossRef] [PubMed]

16. Ewaschuk, J.B.; Unger, S.; Harvey, S.; O'Connor, D.L.; Field, C.J. Effect of pasteurization on immune components of milk: Implications for feeding preterm infants. Appl. Physiol. Nutr. Metab. 2011, 36, 175-182. [CrossRef] [PubMed]

17. Ley, S.H.; Hanley, A.J.; Stone, D.; O'Connor, D.L. Effects of pasteurization on adiponectin and insulin concentrations in donor human milk. Pediatr. Res. 2011, 70, 278-281. [CrossRef] [PubMed]

18. García-Lara, N.R.; Vieco, D.E.; De la Cruz-Bértolo, J.; Lora-Pablos, D.; Velasco, N.U.; Pallás-Alonso, C.R. Effect of Holder pasteurization and frozen storage on macronutrients and energy content of breast milk. J. Pediatr. Gastroenterol. Nutr. 2013, 57, 377-382. [CrossRef] [PubMed]

19. Vieira, A.A.; Soares, F.V.; Pimenta, H.P.; Abranches, A.D.; Moreira, M.E. Analysis of the influence of pasteurization, freezing/thawing, and offer processes on human milk's macronutrient concentrations. Early Hum. Dev. 2011, 87, 577-580. [CrossRef] [PubMed]

20. Koenig, A.; de Albuquerque Diniz, E.M.; Barbosa, S.F.; Vaz, F.A. Immunologic factors in human milk: The effects of gestational age and pasteurization. J. Hum. Lact. 2005, 21, 439-443. [CrossRef] [PubMed]

21. Hamprecht, K.; Maschmann, J.; Müller, D.; Dietz, K.; Besenthal, I.; Goelz, R.; Middeldorp, J.M.; Speer, C.P.; Jahn, G. Cytomegalovirus (CMV) inactivation in breast milk: Reassessment of pasteurization and freeze-thawing. Pediatr. Res. 2004, 56, 529-535. [CrossRef] [PubMed]

22. Silvestre, D.; Ferrer, E.; Gayá, J.; Jareño, E.; Miranda, M.; Muriach, M.; Romero, F.J. Available lysine content in human milk: Stability during manipulation prior to ingestion. Biofactors 2006, 26, 71-79. [CrossRef] [PubMed]

23. Góes, H.C.; Torres, A.G.; Donangelo, C.M.; Trugo, N.M. Nutrient composition of banked human milk in Brazil and influence of processing on zinc distribution in milk fractions. Nutrition 2002, 18, 590-594. [CrossRef]

24. Ford, J.E.; Law, B.A.; Marshall, V.M.E.; Reiter, B. Influence of the heat treatment of human milk on some of its protective constituents. J. Pediatr. 1977, 90, 29-35. [CrossRef]

25. Viazis, S.; Farkas, B.E.; Allen, J.C. Effects of high-pressure processing on immunoglobulin A and lysozyme activity in human milk. J. Hum. Lact. 2007, 23, 253-261. [CrossRef]

26. Contador, R.; Delgado-Adámez, J.; Delgado, F.J.; Cava, R.; Ramírez, R. Effect of thermal pasteurisation or high pressure processing on immunoglobulin and leukocyte contents of human milk. Int. Dairy J. 2013, 32, 1-5. [CrossRef]

27. Permanyer, M.; Castellote, C.; Ramírez-Santana, C.; Audí, C.; Pérez-Cano, F.J.; Castell, M.; López-Sabater, M.C.; Franch, A. Maintenance of breast milk immunoglobulin A after high-pressure processing. J. Dairy Sci. 2010, 93, 877-883. [CrossRef] [PubMed]

28. Liebhaber, M.; Lewiston, N.J.; Asquith, M.T.; Olds-Arroyo, L.; Sunshine, P. Alterations of lymphocytes and of antibody content of human milk after processing. J. Pediatr. 1977, 91, 897-900. [CrossRef]

29. Goldsmith, S.J.; Dickson, J.S.; Barnhart, H.M.; Toledo, R.T.; Eiten-Miller, R.R. IgA, IgG, IgM and lactoferrin contents of human milk during early lactation and the effect of processing and storage. J. Food Prot. 1983, 1, $4-7$. 
30. Evans, T.J.; Ryley, H.C.; Neale, L.M.; Dodge, J.A.; Lewarne, V.M. Effect of storage and heat on antimicrobial proteins in human milk. Arch. Dis. Child. 1978, 53, 239-241. [CrossRef] [PubMed]

31. Espinosa-Martos, I.; Montilla, A.; de Segura, A.G.; Escuder, D.; Bustos, G.; Pallás, C.; Rodríguez, J.M.; Corzo, N.; Fernández, L. Bacteriological, biochemical, and immunological modifications in human colostrum after Holder pasteurisation. J. Pediatr. Gastroenterol. Nutr. 2013, 56, 560-568. [CrossRef] [PubMed]

32. Sousa, S.G.; Santos, M.D.; Fidalgo, L.G.; Delgadillo, I.; Saraiva, J.A. Effect of thermal pasteurisation and high-pressure processing on immunoglobulin content and lysozyme and lactoperoxidase activity in human colostrum. Food Chem. 2014, 151, 79-85. [CrossRef] [PubMed]

33. Baro, C.; Giribaldi, M.; Arslanoglu, S.; Giuffrida, M.G.; Dellavalle, G.; Conti, A.; Tonetto, P.; Biasini, A.; Coscia, A.; Fabris, C.; et al. Effect of two pasteurization methods on the protein content of human milk. Front. Biosci. 2001, 3, 818-829. [CrossRef]

34. Czank, C.; Prime, D.K.; Hartmann, B.; Simmer, K.; Hartmann, P.E. Retention of the immunological proteins of pasteurized human milk in relation to pasteurizer design and practice. Pediatr. Res. 2009, 66, 374-379. [CrossRef] [PubMed]

35. Christen, L.; Lai, C.T.; Hartmann, B.; Hartmann, P.E.; Geddes, D.T. The effect of UV-C pasteurization on bacteriostatic properties and immunological proteins of donor human milk. PLoS ONE 2013, 8. [CrossRef] [PubMed]

36. Gibbs, J.H.; Fisher, C.; Bhattacharya, S.; Goddard, P.; Baum, J.D. Drip breast milk: Its composition, collection and pasteurization. Early Hum. Dev. 1977, 1, 227-245. [CrossRef]

37. Henderson, T.R.; Fay, T.N.; Hamosh, M. Effect of pasteurization on long chain polyunsaturated fatty acid levels and enzyme activities of human milk. J. Pediatr. 1998, 132, 876-878. [CrossRef]

38. Ewaschuk, J.B.; Unger, S.; O'Connor, D.L.; Stone, D.; Harvey, S.; Clandinin, M.T.; Field, C.J. Effect of pasteurization on selected immune components of donated human breast milk. J. Perinatol. 2011, 31, 593-598. [CrossRef] [PubMed]

39. Delgado, F.J.; Cava, R.; Delgado, J.; Ramírez, R. Tocopherols, fatty acids and cytokines content of Holder pasteurised and high-pressure processed human milk. Dairy Sci. Technol. 2014, 94, 145-156. [CrossRef]

40. Untalan, P.B.; Keeney, S.E.; Palkowetz, K.H.; Rivera, A.; Goldman, A.S. Heat susceptibility of interleukin-10 and other cytokines in donor human milk. Breastfeed Med. 2009, 4, 137-144. [CrossRef] [PubMed]

41. Goelz, R.; Hihn, E.; Hamprecht, K.; Dietz, K.; Jahn, G.; Poets, C.; Elmlinger, M. Effects of different CMV heatinactivation-methods on growth factors in human breast milk. Pediatr. Res. 2009, 65, 458-461. [CrossRef] [PubMed]

42. Carratù, B.; Ambruzzi, A.M.; Fedele, E.; Sanzini, E. Human Milk Banking: Influence of different pasteurization temperatures on levels of protein sulphur amino acids and some free amino acids. J. Food Sci. 2005, 70, c373-c375. [CrossRef]

43. Valentine, C.J.; Morrow, B.S.; Fernandez, S.; Gulati, P.; Bartholomew, D.; Long, D.; Welty, S.E.; Morrow, A.L.; Rogers, L.K. Docosahexaenoic acid and amino acid contents in pasteurized donor milk are low for preterm infants. J. Pediatr. 2010, 157, 906-910. [CrossRef] [PubMed]

44. Van Zoeren-Grobben, D.; Schrijver, J.; Van den Berg, H.; Berger, H.M. Human milk vitamin content after pasteurisation, storage, or tube feeding. Arch. Dis. Child. 1987, 62, 161-165. [CrossRef] [PubMed]

45. Goldsmith, S.J.; Eitenmiller, I.R.R.; Toledo, R.T.; Barnhart, H.M. Effects of processing and storage on the water-soluble vitamin content of human milk. J. Food Sci. 1983, 48, 994-995. [CrossRef]

46. Moltó-Puigmartí, C.; Permanyer, M.; Castellote, A.I.; López-Sabater, M.C. Effects of pasteurisation and high-pressure processing on vitamin $C$, tocopherols and fatty acids in mature human milk. Food Chem. 2011, 124, 697-702. [CrossRef]

47. Romeu-Nadal, M.; Castellote, A.I.; Gayà, A.; López-Sabater, M.C. Effect of pasteurisation on ascorbic acid, dehydroascorbic acid, tocopherols and fatty acids in pooled mature human milk. Food Chem. 2008, 107, 434-438. [CrossRef]

48. Oliveira, A.M.M.M.; Marinho, H.A. Determination of Vitamin A in the milk of donor mothers from the human milk bank in Manaus/AM. Effect of processing. Acta Amazon. 2010, 40, 59-64. [CrossRef]

49. Ribeiro, K.D.; Melo, I.L.; Pristo, A.Z.; Dimenstein, R. The effect of processing on the Vitamin A content of human milk. J. Pediatr. 2005, 81, 61-64. [CrossRef]

50. Fidler, N.; Sauerwald, T.U.; Demmelmair, H.; Koletzko, B. Fat content and fatty acid composition of fresh, pasteurized, or sterilized human milk. Adv. Exp. Med. Biol. 2001, 501, 485-495. [PubMed] 
51. Lepri, L.; Del Bubba, M.; Maggini, R.; Donzelli, G.P.; Galvan, P. Effect of pasteurization and storage on some components of pooled human milk. J. Chromatogr. B Biomed. Sci. Appl. 1997, 704, 1-10. [CrossRef]

52. Borgo, L.A.; Cohelho Araujo, W.M.; Conceição, M.H.; Sabioni Resck, I.; Mendonça, M.A. Are fat acids of human milk impacted by pasteurization and freezing? Nutr. Hosp. 2015, 31, 1386-1393.

53. Wardell, J.M.; Hill, C.M.; D'Souza, S.W. Effect of pasteurization and of freezing and thawing human milk on its triglyceride content. Acta Paediatr. Scand. 1981, 70, 467-471. [CrossRef] [PubMed]

54. De Segura, A.G.; Escuder, D.; Montilla, A.; Bustos, G.; Pallás, C.; Fernández, L.; Corzo, N.; Rodríguez, J.M. Heating-induced bacteriological and biochemical modifications in human donor milk after Holder pasteurisation. J. Pediatr. Gastroenterol. Nutr. 2012, 54, 197-203. [CrossRef] [PubMed]

55. Bertino, E.; Coppa, G.V.; Giuliani, F.; Coscia, A.; Gabrielli, O.; Sabatino, G.; Sgarrella, M.; Testa, T.; Zampini, L.; Fabris, C. Effects of Holder pasteurization on human milk oligosaccharides. Int. J. Immunopathol. Pharmacol. 2008, 21, 381-385. [PubMed]

56. Coscia, A.; Peila, C.; Bertino, E.; Coppa, G.V.; Moro, G.E.; Gabrielli, O.; Zampini, L.; Galeazzi, T.; Maccari, F.; Volpi, N. Effect of Holder pasteurisation on human milk glycosaminoglycans. J. Pediatr. Gastroenterol. Nutr. 2015, 60, 127-130. [CrossRef] [PubMed]

57. Contador, R.; Delgado, F.J.; García-Parra, J.; Garrido, M.; Ramírez, R. Volatile profile of breast milk subjected to high-pressure processing or thermal treatment. Food Chem. 2015, 180, 17-24. [CrossRef] [PubMed]

58. Silvestre, D.; Miranda, M.; Muriach, M.; Almansa, I.; Jareno, E.; Romero, F.J. Antioxidant capacity of human milk: Effect of thermal conditions for the pasteurization. Acta Paediatr. 2008, 97, 1070-1074. [CrossRef] [PubMed]

59. Elisia, I.; Kitts, D.D. Quantification of hexanal as an index of lipid oxidation in human milk and association with antioxidant components. J. Clin. Biochem. Nutr. 2011, 49, 147-152. [CrossRef] [PubMed]

60. Mateos-Vivas, M.; Rodríguez-Gonzalo, E.; Domínguez-Álvarez, J.; García-Gómez, D.; Ramírez-Bernabé, R.; Carabias-Martínez, R. Analysis of free nucleotide monophosphates in human milk and effect of pasteurisation or high-pressure processing on their contents by capillary electrophoresis coupled to mass spectrometry. Food Chem. 2015, 174, 348-355. [CrossRef] [PubMed]

61. Ballard, O.; Morrow, A.L. Human milk composition: Nutrients and bioactive factors. Pediatr. Clin. N. Am. 2013, 60, 49-74. [CrossRef] [PubMed]

62. Lönnerdal, B. Nutritional and physiologic significance of human milk proteins. Am. J. Clin. Nutr. 2003, 77, 1537S-1543S. [PubMed]

63. Ochoa, T.J.; Cleary, T.G. Effect of lactoferrin on enteric pathogens. Biochimie 2009, 91, 30-34. [CrossRef] [PubMed]

64. Mayayo, C.; Monteserrat, M.; Ramos, S.J.; Martínez-Lorenzo, C.; Calvo, M.; Sánchez, L.; Péreza, M.D. Kinetic parameters for high-pressure-induced denaturation of lactoferrin in human milk. Int. Dairy J. 2014, 39, 246-252. [CrossRef]

65. Hamosh, M. Digestion of the premature infant: The effect of the human milk. Sem. Perinatol. 1994, 18, 485-494.

66. Macdonald, L.E.; Brett, J.; Kelton, D.; Majowicz, S.E.; Snedeker, K.; Sargeant, J.M. A systematic review and meta-analysis of the effects of pasteurization on milk vitamins, and evidence for raw milk consumption and other health-related outcomes. J. Food Prot. 2011, 74, 1814-1832. [CrossRef] [PubMed]

67. Newburg, D.S. Glycobiology of human milk. Biochemistry 2013, 78, 771-785. [CrossRef] [PubMed]

(C) 2016 by the authors; licensee MDPI, Basel, Switzerland. This article is an open access article distributed under the terms and conditions of the Creative Commons Attribution (CC-BY) license (http://creativecommons.org/licenses/by/4.0/). 